\title{
CIFPSS 2021 : un congrès singulier
}

\section{CIFPSS 2021: a singular congress}

Le prochain Congrès international francophone de pédagogie des sciences de la santé (CIFPSS) sera singulier.

Singulier, d'abord, en raison de la situation sanitaire, qui mettra transitoirement les intervenants et les participants à distance les un des autres, et qui impliquera que les interactions soient médiatisées par des outils numériques devenus, le temps d'une année qui a trop duré, le partenaire privilégié des organisateurs de congrès.

Singulier, ensuite, parce qu'il témoigne d'une dynamique inédite du champ de l'éducation des sciences de la santé dans le monde francophone, tel qu'en témoigne le nombre record de soumissions -240 ateliers et communications libres - expertisées par un comité scientifique international et pluriprofessionnel.

Singulier, aussi, par le format, principalement constitué d'activités d'apprentissage en groupes (les ateliers, les tables rondes et les débats), et dont les conférences plénières prendront la forme de courtes interventions ciblées sur les préoccupations actuelles des formateurs et enseignants en santé ( $\ll 10$ minutes pour avoir les idées claires sur le concept de [...]»), tout en accordant une place de choix à des communications orales qui se distinguent par leur intérêt et leur qualité exceptionnels («Une idée qui vaut la peine d'être partagée»), et en mettant au premier plan la science et l'éducation fondée sur les preuves, grâce aux chercheurs qui ont accepté de partager «Une publication [de leur choix] qui pourrait changer la vie des formateurs et des enseignants ».

Singulier, encore, par la parole donnée aux derniers maillons de la chaîne, celui des bénéficiaires des formations en santé - les étudiants et les patients-, qui animeront la séance d'ouverture du congrès en s'adressant aux formateurs, aux enseignants et aux responsables institutionnels.

Singulier, toujours, en raison de la période, marquée par la mise en œeuvre de réformes de grande ampleur, parmi lesquelles l'universitarisation de l'ensemble des formations en santé, l'accès aux fonctions universitaires des enseignants issus des filières maïeutique, sciences infirmières et sciences de la rééducation, ou encore le déploiement des réformes du premier et du deuxième cycles des études de médecine.
Singulier, enfin, par la ville qui accueillera le congrès - Strasbourg -, haut lieu de la pédagogie universitaire, où plusieurs personnalités occupent des responsabilités au sein d'instances et sociétés savantes nationales et internationales impliquées dans la formation des étudiants en sciences de la santé, et qui héberge le Centre de formation et de recherche en pédagogie des sciences de la santé (CFRPS), entité unique en France quant à son engagement dans la recherche en pédagogie et sur les thématiques $\mathrm{du}$ raisonnement clinique et de la prise de décisions - fil rouge de toutes les réformes des études de santé-, et dans la formation pédagogique des étudiants et des professionnels de la santé.

Bernard CHARLIN
Président de la SIFEM
Anne DEMEESTER
Présidente du comité d'organisation du CIFPSS
Jean JOUQUAN
Rédacteur en chef de Pédagogie Médicale
Thierry PELACCIA
Président du comité Scientifique du CIFPSS
pelaccia@unistra.fr

Jean SIBILIA, ancien président de la Conférence des doyens de médecine et doyen de la faculté de médecine, maïeutique et sciences de la santé de Strasbourg, adresse aux congressistes et à notre communauté un message plein d'espoir, tourné vers l'avenir.

Le congrès de la Société internationale francophone d'éducation médicale (SIFEM) est un événement incontournable pour nos facultés, nos équipes pédagogiques et nos étudiants. Cet événement est l'occasion de réaffirmer notre volonté de faire de la formation médicale une priorité pour mieux préparer nos jeunes aux immenses enjeux de demain.

Pour que nous réussissions ensemble la transformation souhaitée de la formation médicale, il faut que nous partagions quelques fondamentaux déterminants:

- Cette formation ne devra pas être exclusivement médicale, mais elle devra concerner tous les soignants avec une vision nouvelle «centrée»sur le patient et, demain, la prise en compte du sujet «sain» dans une stratégie préventive, prédictive et personnalisée; 
- Cette formation doit inclure comme fil rouge des notions majeures comme celle de la pertinence des soins et des actes, et celle de l'éthique et de la déontologie de nos pratiques qui se dématérialisent progressivement;

- Cette formation doit intégrer une réflexion pédagogique innovante qui a pour objectif une compréhension des connaissances nouvelles, mais aussi le développement de compétences, de 《savoir-faire» et 《savoir-être» des soignants, avec des objectifs de terrain très pratiques;

- Cette formation doit prendre en compte et anticiper l'évolution de la médecine et de ses outils numériques pour préparer les jeunes à une médecine connectée et une coopération avec une intelligence artificielle qui viendra en complément de l'intelligence naturelle des soignants. C'est un enjeu majeur qu'il faut préparer par de nouveaux cursus de formation;

- Cette formation doit transmettre une prise de conscience des grands enjeux que sont le handicap, la précarité, la lutte contre l'intolérance et, surtout, les risques environnementaux;

- Cette formation doit aussi intégrer la notion de bien-être au travail, avec des moments d'ouverture et des échanges internationaux plus nombreux. Il faut dynamiser et faire «respirer» les études de santé, ce qui ne pourra que renforcer la fibre humaniste de nos étudiants ;

- Cette formation doit in fine faire prendre conscience, avec une forme de hauteur de vue, de ce qu'est la santé des hommes. À l'image de la pensée de Georges Canguilhem, nous devons réfléchir à ce qu'est une maladie, sans oublier que cela dépend fondamentalement de la société et du système dans lequel nous vivons. Cependant, quels que soient la culture, le niveau socioéconomique ou l'enjeu éthique, l'OMS avait déjà, en 1946, défini la santé comme un état de bien-être physique, psychique et social, ce qui est et reste une définition universelle.

Ainsi, notre responsabilité sociale est engagée pour faire de la formation en santé une priorité qui réponde aux besoins des hommes et de nos sociétés, car les trois piliers de la cohésion sociale et du bien-être sont la santé, l'éducation et la justice. Ces fondamentaux exigent de la solidarité, de la générosité et du respect mutuel, ce qui est aussi, dans toutes les cultures, le socle du sens de la vie.

Le congrès de la SIFEM va être un lieu d'échanges, véritable agora de la pédagogie. Nous vous attendons nombreux pour des conférences - comme celle sur les grands mythes de la pédagogie - des tables rondes, des ateliers et des présentations scientifiques de projets de recherche. Nous échangerons dans le grand monde de la francophonie, avec convivialité et conviction, pour construire une pédagogie des sciences de la santé innovante et ambitieuse.

Merci à tous ceux qui seront à nos côtés et merci à tous ceux qui donnent avec tant de générosité leur énergie pour faire avancer les choses. Cultivons ensemble ce formidable état d'esprit qui va si bien à la pédagogie des sciences de la santé.

Jean SIBILIA Président d'honneur du CIFPSS 